\title{
Terahertz heterodyne imager for biomedical applications
}

Peter H. Siegel, Robert J. Dengler

Peter H. Siegel, Robert J. Dengler, "Terahertz heterodyne imager for biomedical applications," Proc. SPIE 5354, Terahertz and Gigahertz Electronics and Photonics III, (8 April 2004); doi: 10.1117/12.540027

SPIE Event: Integrated Optoelectronic Devices 2004, 2004, San Jose, CA, United States 
Invited Paper

\title{
Terahertz Heterodyne Imager for Biomedical Applications
}

\author{
Peter H. Siegel and Robert J. Dengler \\ California Institute of Technology Jet Propulsion Laboratory, Pasadena, CA 91109
}

\section{INTRODUCTION}

A wealth of useful spectral information exists at submillimeter-wave frequencies: $300 \mathrm{GHz}-3000 \mathrm{GHz}$ (wavelengths from $1 \mathrm{~mm}$ to 100 microns), and astronomers have long pursued programs to develop this technology for Earth, planetary and space science applications [1]. Over the past three years there has been an explosion of interest in this wavelength range in the biological and biomedical areas [2,3]. However the very properties that make this frequency regime so interesting-high absorption from many gaseous species, liquids and solids, especially water (Fig. 1)-make it extremely difficult for significant penetration or propagation of terahertz energy, thus severely limiting imaging, radar or communications applications. Nevertheless, significant progress has been made in the examination of biological and other material samples through the use of ultra-fast-pulse time domain spectroscopy (TDS) $[4,5]$. This technique yields wide spectral coverage but has limited frequency resolution and signal-to-noise ratio (limited penetration depth). As a contrast to TDS, CW heterodyne imaging [6] is an old technique that offers the potential for extremely large dynamic range and high signal-to-noise ratio while maintaining fast data acquisition, stable magnitude and phase measurements, reasonable frequency flexibility and $\mathrm{mm}$ scale penetration in wet tissues and other biomaterials.

This paper describes a CW terahertz heterodyne imaging system based around two custom fabricated $2.5 \mathrm{THz}$ planar Schottky diode mixers [7] and two commercially available optically pumped far IR lasers [8]. One laser is used for the signal beam and supplies as much as $70 \mathrm{~mW}$ at $2.5 \mathrm{THz}$. The other laser acts as a local oscillator (LO) source for the two mixers. Line pairs very close to each other $\left(\mathrm{CH}_{3} \mathrm{OH}\right.$ and $\left.\mathrm{CH}_{2} \mathrm{~F}_{2}\right)$ are chosen to provide a workable intermediate frequency output (IF) of $24 \mathrm{GHz}$. Broader RF bandwidth is possible with tunable signal sources and wider IF band amplifiers. A novel frequency stabilization scheme has been implemented to track and calibrate the laser power (magnitude and phase) over a sample run. The system uses the second $\mathrm{THz}$ mixer, a low frequency $(\mathrm{GHz})$ reference oscillator and a lock-in amplifier to monitor and normalize the two lasers (LO and signal). Stability of $\sim 0.1 \mathrm{~dB}$ and $<5$ degrees have been achieved with a dynamic range of more than $100 \mathrm{~dB}$. The present system scans the sample through the focused beam and measures transmission or reflection at a fixed RF frequency. Penetration in wet (saline or formalin perfused) tissue is more than $2 \mathrm{~mm}$ at $2.5 \mathrm{THz}$ (contrasted with microns in a TDS or FTS system at this wavelength). Applications to date include establishing contrast mechanisms in a range of biological and electronic materials. Future applications include fast 2D and 3D transmission/reflection imaging, pump-probe resonance in liquids and solids, radiation induced effects in biomaterials, change of state measurements, and radiometric imaging of subjects in-vivo.

\section{DESCRIPTION OF THE TEST SYSTEM}

When considering an investigation of the transmission properties of biomaterials at terahertz frequencies, it is imperative to realize that the associated losses due to water absorption are untenably high $\left(\mathrm{I}=\mathrm{I}_{0} \mathrm{e}^{-\alpha \mathrm{x}}\right.$, where $\mathrm{x}$ is in $\mathrm{cm}$ and $\alpha$ is over $450 \mathrm{~cm}^{-1}$ at $2.5 \mathrm{THz}$ (Fig. 2). Although this absorption can provide significant differential contrast, it comes at a great price - very shallow penetration in saline or formalin perfused tissue and many other biomaterials. An imaging system that can measure weak signals through very lossy samples is desirable. In this frequency domain, such a system can be constructed using well-known $\mathrm{CW}$ heterodyne and radiometric techniques if a strong $\mathrm{THz}$ illumination signal and close frequency spaced local oscillator source are available. The recent introduction of commercial $\mathrm{CO}_{2}$ pumped far IR lasers [8] and high sensitivity room temperature THz planar Schottky diode downconverters [7] makes such a THz heterodyne imaging system realizable. In order to achieve an optimal signal-to-noise ratio $(\mathrm{S} / \mathrm{N})$, the pre-detection bandwidth must be made as narrow as possible. However, in order to obtain an image of reasonable resolution (many scanned pixels), it is desirable to use the largest possible pre-detection bandwidth (maximum signal strength). In addition to these conflicting requirements, there is the added complication of the stability of the THz sources (better than 1 part in $10^{9}$ is 
required, i.e. $3 \mathrm{kHz}$ ). The minimum frequency drift of the commercial $\mathrm{CO}_{2}$ pumped far IR lasers, over a 5 minute period, is about $150 \mathrm{kHz}$. Bearing these limitations in mind and taking advantage of space sensor technology that we have developed for several NASA missions, a system for imaging the transmission loss and phase of thin $(<10 \mathrm{~mm}$ thick $)$ biosamples at $2.523 \mathrm{THz}$ has been constructed and successfully tested in the laboratory. Dynamic range as great as 100 $\mathrm{dB}$ has been measured to date with a $3 \mathrm{~dB}$ pixel size of $<0.4 \mathrm{~mm}$ (this can be improved with different optics). The noise in the phase measurements is less than $+/-5$ degrees.

Before implementing the heterodyne system, our sample scanning and image acquisition and display algorithms were verified with the simple fixed-frequency direct detection scheme shown in Fig. 3. This configuration employs a heliumcooled commercial silicon microbolometer [9] for the detection of the transmitted $\mathrm{THz}$ signal which is generated by a Coherent-DEOS SIFIR-50 $\mathrm{CO}_{2}$-pumped molecular vapor gas laser operated on the $118.83 \mu \mathrm{m}$ line of methanol, $\mathrm{CH}_{3} \mathrm{OH}$. Output power from the laser peaks at about $70 \mathrm{~mW}$. The $\mathrm{THz}$ beam is focused and passed through the test sample, then refocused into the helium-cooled detector which saturates at power levels above a few microwatts. The $\mathrm{THz}$ signal is mechanically chopped and detected by a standard lock-in amplifier as the sample is scanned through the beam. A dynamic range of about $40 \mathrm{~dB}$ was achieved with a tissue penetration slightly less than $0.5 \mathrm{~mm}$, amplitude stability near $0.2 \mathrm{~dB}$, spatial resolution of $0.5 \mathrm{~mm}$ and a data acquisition time of approximately 10 pixels $/ \mathrm{sec}$. Typical images obtained with this direct detection system are shown in the figure. Only the amplitude of the transmitted power can be measured with this arrangement.

Our first realization of a true $\mathrm{CW} \mathrm{THz}$ heterodyne imaging system is shown in Fig. 4. It consists of two DEOS lasers both operated on the $118.83 \mu \mathrm{m} \mathrm{CH}_{3} \mathrm{OH}$ line feeding an in-house developed $2.5 \mathrm{THz}$ planar GaAs Schottky diode mixer. The local oscillator (LO) laser is fed straight into the mixer while the signal (RF) laser beam is focused down from its nominal $3 \mathrm{~mm}$ diameter to $\sim 1 \mathrm{~mm}$ at the sample plane using a pair of Teflon lenses. The sample is moved in a plane normal to the beam using a computer controlled X-Y stage. Slight offsets applied to both methanol lasers provided about a $2.5 \mathrm{MHz}$ signal at the mixer IF output. This IF is fed to an Agilent E4408 spectrum analyzer, which serves as the power detector. Because of the poor $\mathrm{S} / \mathrm{N}$ at $1 \mathrm{MHz}$ resolution (pre-detection) bandwidth, the bandwidth is set to a value much lower than the long-term drift of the lasers $(>150 \mathrm{kHz})$ and the power is read using the "peak find" feature of the analyzer. Bugs in the E4408 firmware severely limit the maximum speed of the system to that at which the analyzer can report the peak power in the trace. With the simple heterodyne system shown in the figure a dynamic range of about $60 \mathrm{~dB}$ can be obtained giving a penetration depth of $1 \mathrm{~mm}$ in tissue, amplitude resolution of approximately $0.2 \mathrm{~dB}$, and an acquisition rate of 5 pixels per second (limited by the E4408). Due to instabilities in frequency no phase information is obtained.

Greatly improved dynamic range and frequency stability can be obtained with the more complex arrangement shown in Fig. 5. whereby the IF signal to be detected is essentially phase stabilized against itself. At the suggestion of Dr. Eric Mueller of Coherent-DEOS, a phase stabilization system patterned after Doane [10] was constructed using two THz downconverters. The signal from the DEOS far IR laser is split into measurement and reference paths and is mixed in each downconverter using the same LO signal (derived from the second far IR laser). This results in two IF signals that are equal in instantaneous frequency but differ in amplitude and phase. By further mixing the reference IF with a 3.2 $\mathrm{GHz}$ oscillator, filtering and amplifying the result, and applying it to the LO input of another mixer to downconvert the measurement IF, a 2nd IF signal is obtained that is equal in instantaneous frequency to the $3.2 \mathrm{GHz}$ oscillator (hence very narrow linewidth) but differs in amplitude and phase depending on the loss and phase shift imposed on the signal beam by the scanned sample. In order to maximize throughput, the spectrum analyzer was replaced with a Stanford Research SR844 RF lock-in amplifier. Since the lock-in amplifier has a maximum operating frequency of $200 \mathrm{MHz}$ with peak performance below $10 \mathrm{MHz}$, the two $3.2 \mathrm{GHz}$ signals were downconverted to approximately $9 \mathrm{MHz}$ using a common, highly-isolated oscillator to feed both mixers. In addition to having a maximum two millisecond latency between the issuance of a trigger command to it and the actual logging of data, the lock-in amplifier can also measure the relative phase difference between the reference and measurement IF signals, thereby providing phase delay imagery of the samples. In order to improve the spatial resolution of the imaging system and reduce optics losses by $\sim 20 \mathrm{~dB}$, the Teflon lenses were replaced with a pair of off-axis parabolic mirrors. This change reduced the beam diameter to $<0.4$ $\mathrm{mm}$.

The original tracking circuit described in Doane [10] specified the use of isolators at the RF inputs of the mixers to reduce the amount of signal reflected off of the mixer inputs. Unfortunately, such isolators are not available at $2.5 \mathrm{THz}$. 
Therefore, limited measurement range was expected due to some RF laser power bypassing the sample plane via the reference mixer input and then appearing at the measurement mixer input. Initially, the dynamic range of the system was only $45 \mathrm{~dB}$. A $2.5 \mathrm{THz}$ isolator consisting of a quarter-wave plate and polarizing grid was placed on the LO laser output in an attempt to prevent back-reflected RF laser power from the reference mixer from propagating down into, and then back out of, the LO laser to the measurement mixer, but this was ineffective. However, due to the large amount of available power at the lasers $(\sim 60 \mathrm{~mW})$ and the excellent performance of the $\mathrm{THz}$ mixers, it was found that attenuation in the form of small paper sheets $(\sim 5 \mathrm{~dB}$ per sheet) could be added in front of the reference mixer and LO laser to provide effective isolation without significantly degrading the performance of the measurement mixer. By changing the lasing medium of one of the lasers to difluoromethane in order to output a laser line frequency $23.7 \mathrm{GHz}$ higher than the $\mathrm{CH}_{3} \mathrm{OH}$ signal, it is possible to use a frequency diplexer in the beam. Using specially manufactured etalons made from high-resistivity silicon [11] (see Fig. 6), an additional 40 to $50 \mathrm{~dB}$ of isolation between the two $\mathrm{THz}$ mixers was obtained. This isolation is a result of the extremely low reflectivity $(<-25 \mathrm{~dB})$ of the etalons at $2.523 \mathrm{THz}$ when the angle of incidence is optimized. Block diagrams of the complete RF and IF systems are shown in Figs. 6 and 7 and some initial images from this test set are shown in Figs. 8 and 9. More than $10^{10}(100 \mathrm{~dB})$ of dynamic range has been realized.

Operation at frequencies other than $2.5 \mathrm{THz}$ is possible by judicious choice of laser lines and replacement of the two Schottky diode mixers with downconverters optimized for the different frequencies. Sensitivity enhancement is also possible (at the expense of high-end dynamic range) by replacing the Schottky mixers with more sensitive hot electron bolometer [12] or superconductor-insulator-superconductor [13] elements. Factors of 10-100 in low-end signal acquisition are possible with these near quantum limited receiver elements. Finally, significant improvement in frequency agility might soon be achieved by replacing the two far IR lasers with recently available semiconductor quantum cascade lasers [14].

\section{CONCLUSION}

A heterodyne imaging system has been constructed that can sample the magnitude and phase of transmitted or reflected power off of mechanically scanned samples at $\mathrm{THz}$ frequencies. The system has a measured dynamic range of over 100 $\mathrm{dB}, 0.1 \mathrm{~dB}$ amplitude stability, 5 degrees of phase resolution and can sample $>100$ pixels/second (limited only by the translation stage). Applications are just beginning to materialize and include a systematic study of contrast mechanisms in biological samples, pump-probe excitation of phonon modes in liquids and solids, RF irradiance effects on cellular function and morphology, and true radiometric measurements of animal subjects in-vivo. As far as the authors know there are no other systems of this design currently in use. Competing techniques for $\mathrm{THz}$ imaging utilize short pulse time domain spectroscopy and Fourier transform spectroscopy (FTS), both extremely powerful methodologies but with limitations on dynamic range and spectral resolution. Although the $\mathrm{THz}$ imaging system described in this paper cannot hope to duplicate the spectroscopic coverage of the TDS and FTS systems, it can enhance narrow band measurements and provide an alternative imaging technology with complementary, and in some cases (wherever strong CW signals might be useful), unique function. Commercial interest in this system will be stimulated by reduced size (replacement of the laser signal source and LO with solid-state units when they eventually become available [15]), electronic frequency hopping or sweeping (available with multiplier and photomixer sources [16] and perhaps soon with quantum cascade lasers [14]), and movable transmitter/receiver heads (using flexible THz transmission media [17]).

\section{ACKNOWLEDGEMENTS}

The authors gratefully acknowledge the support of Dr. Warren S. Grundfest (UCLA) and Prof. Scott E. Fraser (Caltech Biological Imaging Center). They would also like to acknowledge the continued interest and frequent help of Dr. Eric Mueller at Coherent DEOS, who first suggested the amplitude tracking laser scheme. Dr. Carol Readhead and Mr. Tim Hiltner, both at Caltech, have been a huge help in providing the mouse samples and training for the biological prep work. Members of the Submillimeter Wave Advanced Technology (SWAT) team at JPL also deserve mention for continuing program and project support. This work was performed at the Jet Propulsion Laboratory, California Institute of Technology under contract with the National Aeronautics and Space Administration and at the California Institute of Technology, Beckman Institute, Division of Biology. Funding was supplied jointly by NASA Code R and NIH through grant K25 EB00109-02. 


\section{REFERENCES}

[1]. P. H. Siegel, “Terahertz Technology,” IEEE Trans. Microwave Theory and Techniques, Special 50th Anniversary Issue, vol. MTT-50, no. 3, March 2002.

[2]. M. Chamberlain and M. Smith, editors, "Proc. of the First International Conf. on Biomedical Imaging and Sensing Applications of THz Technology," Physics in Medicine and Biology, vol. 47., no. 21, pp. 3667-3892, Nov. 7, 2002.

[3]. G.P. Gallerano, M. Scarfi, W. Mantele, editors, "Proceedings of the THz-Bridge Workshop, Palazzo dei Congressi, Capri, Italy, Sept. 29- Oct. 2, 2002,”Journal of Biological Physics, vol. 29, nos. 2-3, pp. 77-338, 2003.

[4]. X-C. Zhang, "T-Ray Sensing and Imaging," in Terahertz Sensing Technology, Volume 1: Electronic Devices and Advanced Systems, Editors: D.L. Woolard, et.al., World Scientific Publishing Co. Pte., Ltd., Singapore, 2003.

[5]. D. Mittleman, "Terahertz Imaging," in Sensing with Terahertz Radiation, editor: D. Mittelman, Springer Series in Optical Sciences, Springer-Verlag, Berlin, 2003.

[6]. Peter H. Siegel, Robert J. Dengler, Eric Mueller, Carol Readhead and Scott Fraser, "Scanned-Pixel Terahertz Heterodyne Imaging,” THz Gap: Meeting of the British Royal Society, London, UK, June 5, 2003.

[7]. P.H. Siegel, R.P. Smith, S. Martin and M. Gaidis, “2.5 THz GaAs Monolithic Membrane-Diode Mixer,” IEEE Transactions Microwave Theory and Techniques, vol. 47, no. 5, pp. 596-604, May 1999.

[8]. E.R. Mueller, W.E. Robotham, Jr., R.P. Meisner, R.A. Hart, J. Kennedy and L.A. Newman, "2.5 THz laser local oscillator for the EOS Chem I satellite," Proc. 9th. Int. Conf. on Space THz Tech, p.563, Pasadena, CA, March 1998.

[9]. Infrared Laboratory HDL-5 silicon bolometer, 1808 E. 17th St., Tucson, AZ, 85719.

[10]. J. L. Doane, "Broadband superheterodyne tracking circuits for millimeter-wave measurements," J. L. Doane, Rev. Sci. Instr., vol. 51, no. 3, pp. 317-20, March 1980.

[11]. The etalons were made from float zone silicon wafers $(>20,000 \mathrm{ohm}-\mathrm{cm})$ available from Topsil Semiconductor Inc., Frederikssund, Denmark and then lapped to their required thickness ( 387.5 and 391.1 microns) by Boston Piezo Optics, Inc. Bellingham, MA.

[12]. W.R. McGrath, B.S. Karasik, A. Skalare, B. Bumble and H.G. LeDuc, "Hot electron superconductive mixers for THz frequencies,” SPIE Terahertz Spectroscopy and Applications conference, vol. 3617, pp. 80-88, Jan. 1999.

[13]. A. Karpov, D. Miller, F. Rice, J. Zmuidzinas, J. Stern, B. Bumble and H.G. LeDuc, "Low noise 1.2 THz SIS receiver," 8th Int. Superconducting Electronics Conference, Osaka, Japan, pp. 521-522, June 19-22, 2001.

[14]. A. Barkan, D. Mittleman, R. Dengler, P.H. Siegel, J. Faust, "Measurement of the linewidth of a Terahertz Quantum Cascade Laser,” CLEO/QELS Conference, Baltimore, MD, June 1-6, 2003.

[15]. Imran Mehdi, E. Schlecht, G. Chattopadhyay and P.H. Siegel, "THz Local Oscillator Sources: Performance and Capabilities," Astronomical Telescopes and Instrumentation, Waikoloa, Hawaii, August 22-28, 2002.

[16]. E.R. Brown, K. McIntosh, K. Nichols and C. Dennis, "Photomixing up to $3.8 \mathrm{THz}$ in low-temperature-grown GaAs,” Applied Phys. Lett., vol. 66, no. 3, pp. 285-87, Jan. 1995.

[17]. C. Yeh, D.L. Rascoe, F. Shimabukuro, M. Tope and P.H. Siegel, "Millimeter and Submillimeter Wave Integrated Circuits based on Polymer Coated Alumina, Si, GaAs or InP Ribbons," JPL New Tech. Rpt., NPO 30339, Aug. 2001. 


\section{FIGURES}

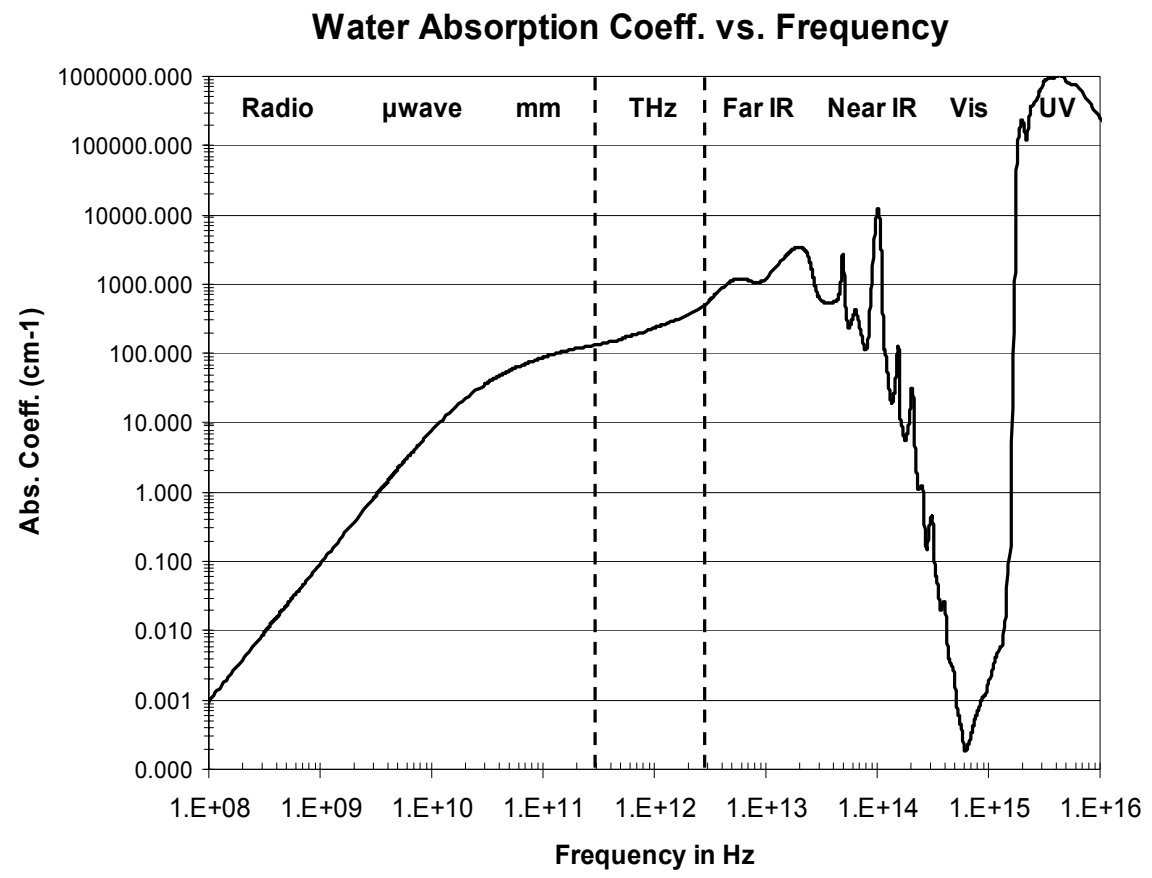

Fig. 1. Absorption coefficient of triply de-ionized water at 292K between $100 \mathrm{MHz}$ and the UV. Reprinted from D.J. Segelstein, MS Thesis, U. Missouri, Kansas City, 1981.

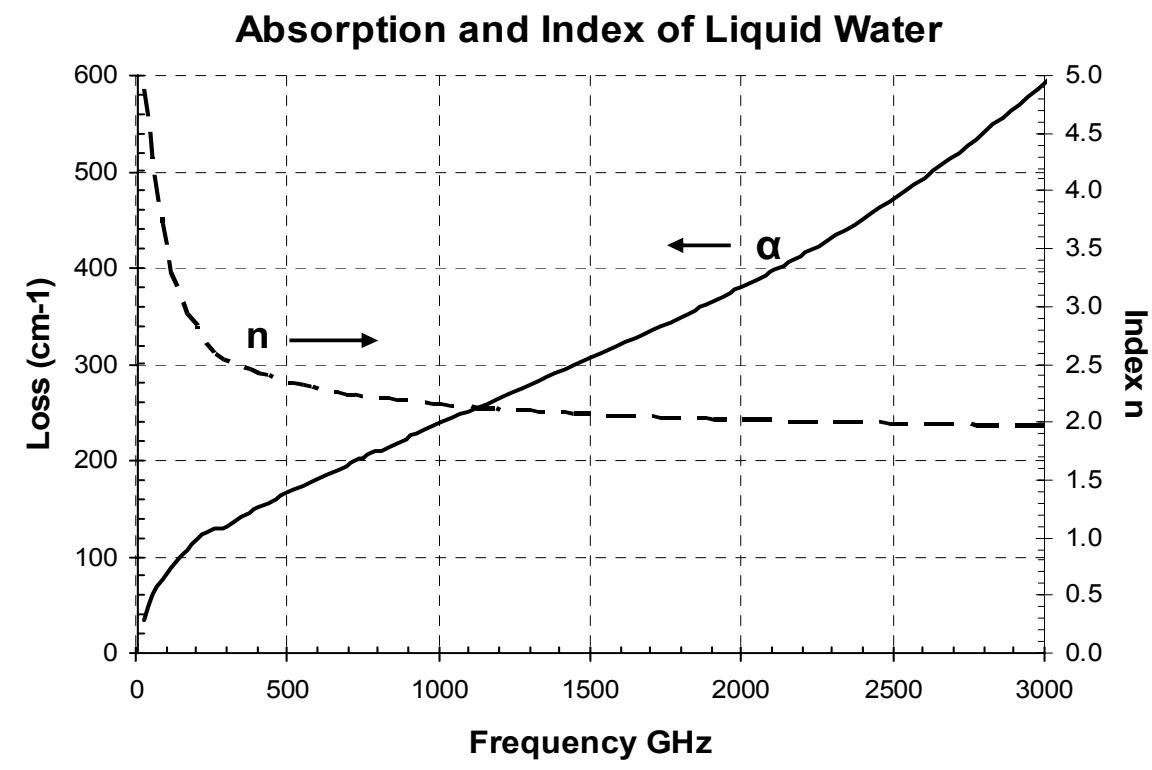

Fig. 2. Absorption coefficient and index of refraction for de-ionized water between 100 and $3000 \mathrm{GHz}$. Reprinted using data available from John Bertie Univ. of Alberta, Canada, at: http://www.ualberta.ca/ jbertie/JBDownload.HTM\#Spectra or published as: J.E. Bertie and Z.D. Lan, "Infrared intensities of liquids: The intensity of the OH stretching band of liquid water revisited, and the best current values of the optical constants of $\mathrm{H}_{2} \mathrm{O}$ at 25 degrees $\mathrm{C}$ between 15,000 and $1 \mathrm{~cm}^{-1}$," Applied Spectroscopy, vol. 50, no.8, pp. 1047-1057, Aug. 1996. 


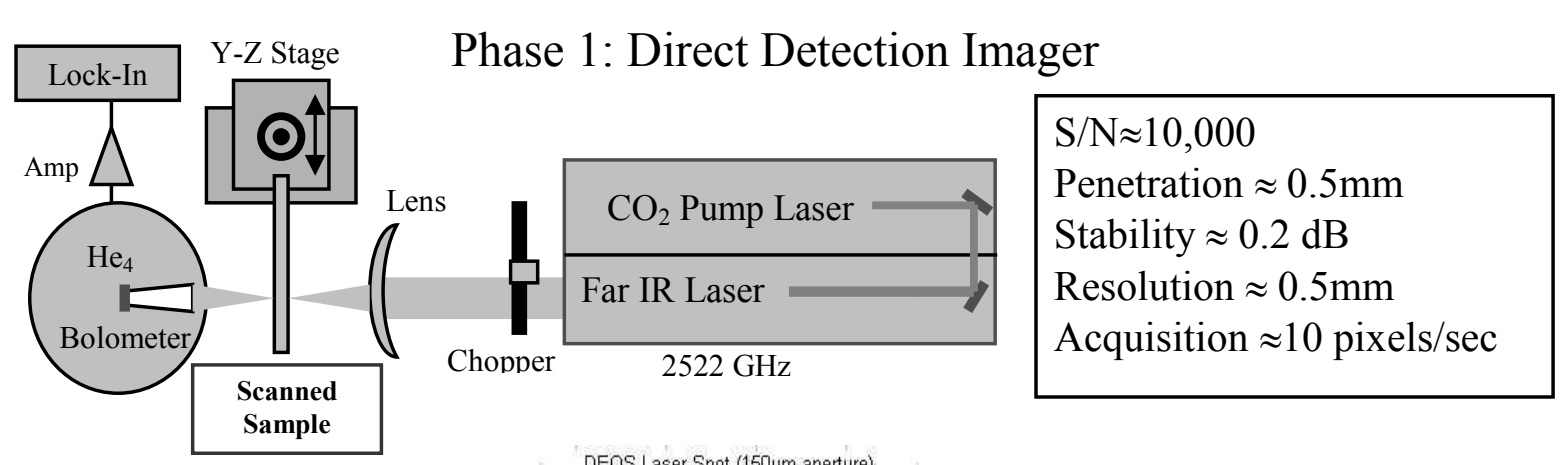

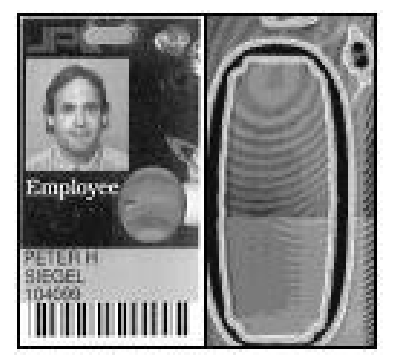

Optical Image THz Image

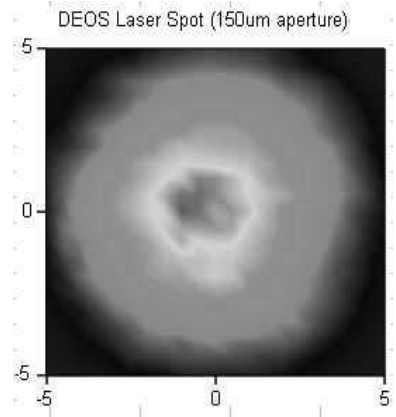

Image of $2.5 \mathrm{THz}$ Laser Beam

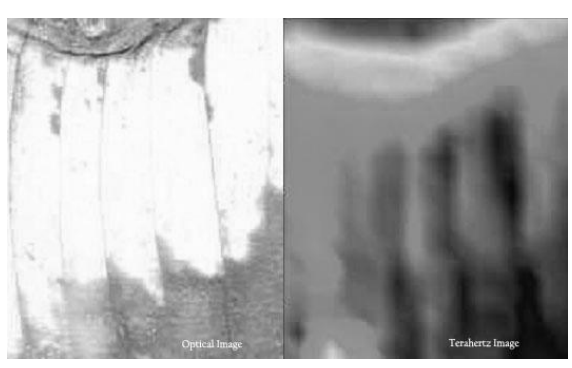

Optical Image THz Trans. Image

Fig. 3. First realization of THz imager using a direct detector helium cooled bolometer and high power far IR laser source. The signal frequency is determined by the laser and has a stability of several hundred $\mathrm{kHz}$ centered at 2.5 THz. A signal-to-noise ratio of about 10,000 was achievable.

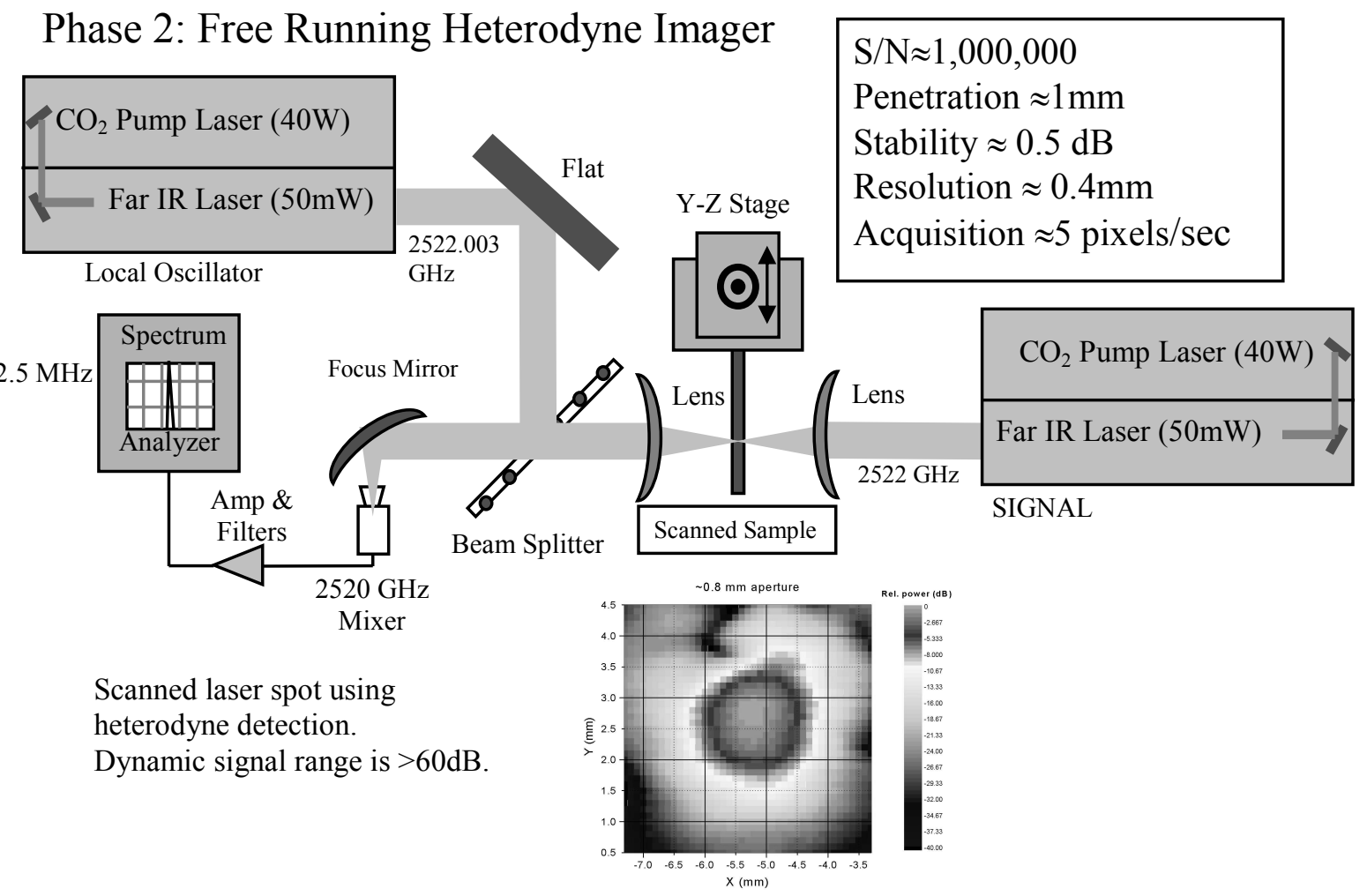

Fig. 4. Initial realization of a heterodyne THz imager using two FIR lasers and a Schottky diode mixer. The lasers are detuned slightly to generate an IF of about $2.5 \mathrm{MHz}$. Operational parameters are shown in the insert. 


\section{Phase 3: Low IF Amplitude and Phase Stabilized Heterodyne Imager}

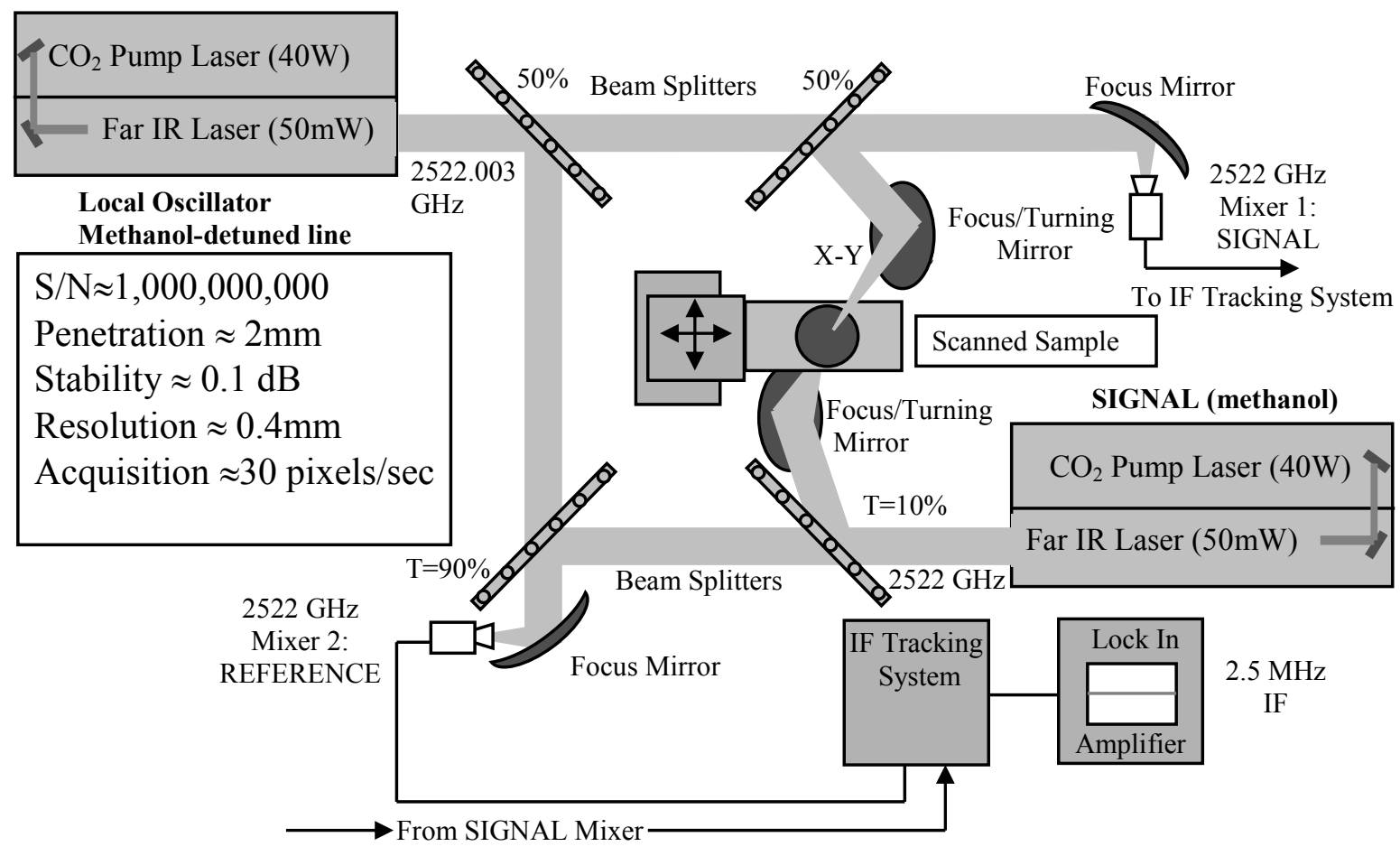

\section{Formalin Perfused Mouse brain sections 2 mm thick, visible and THz images}

Brain sections courtesy Dr. Carol Readhead and Mr. Tim Hiltner, Caltech, BIC.
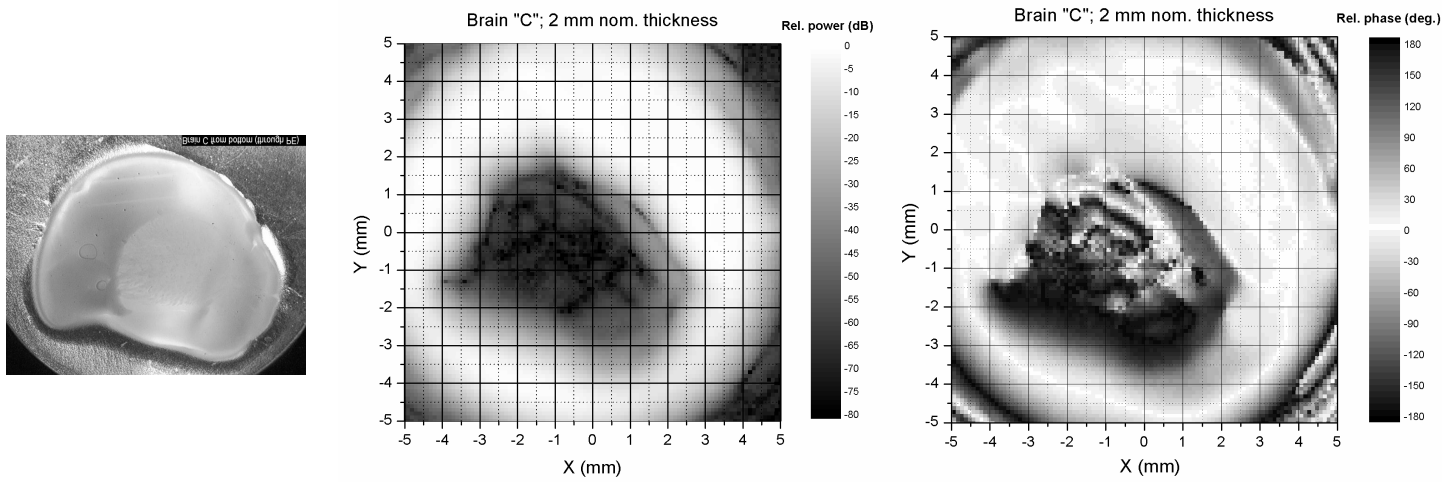

Fig. 5. TOP: Frequency stabilized heterodyne imaging system using two mixers driven by the same LO. Again the IF is generated by a slight detuning of the pump laser system for an IF of 1-3 MHz. Dynamic range is enhanced to $10^{9}$ and acquisition time is now limited by the speed of the X-Y stage. BOTTOM: Visible (left), magnitude (middle) and phase (right) pictures showing penetration of a $2 \mathrm{~mm}$ sample of mouse brain tissue at $2.5 \mathrm{THz}$. 


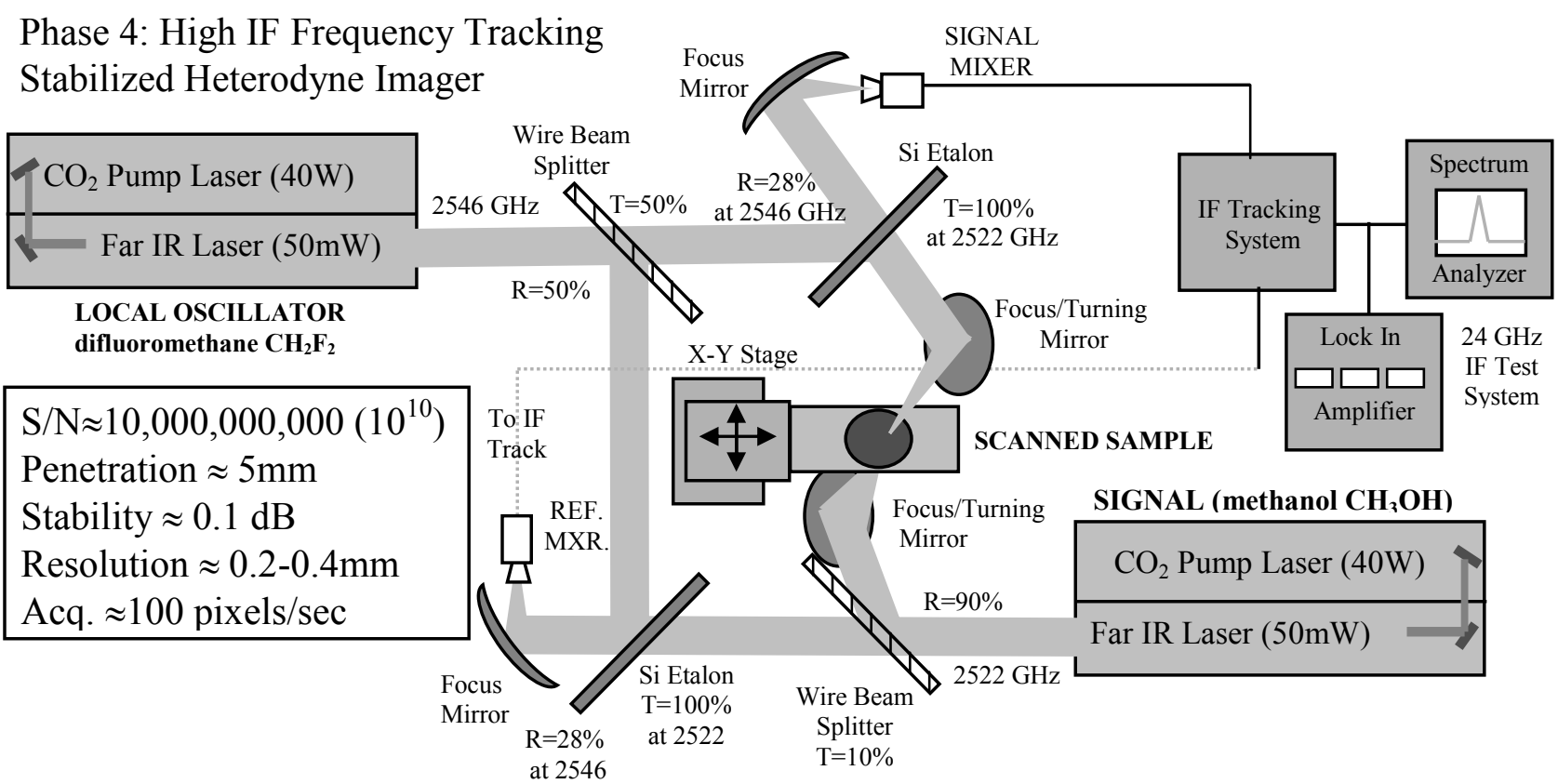

Fig. 6. Frequency-stabilized heterodyne imaging system with the signal and pump lasers operating on two closely spaced $(24 \mathrm{GHz})$ lines: methanol and difluoromethane. The high IF allows greater frequency stability and S/N.

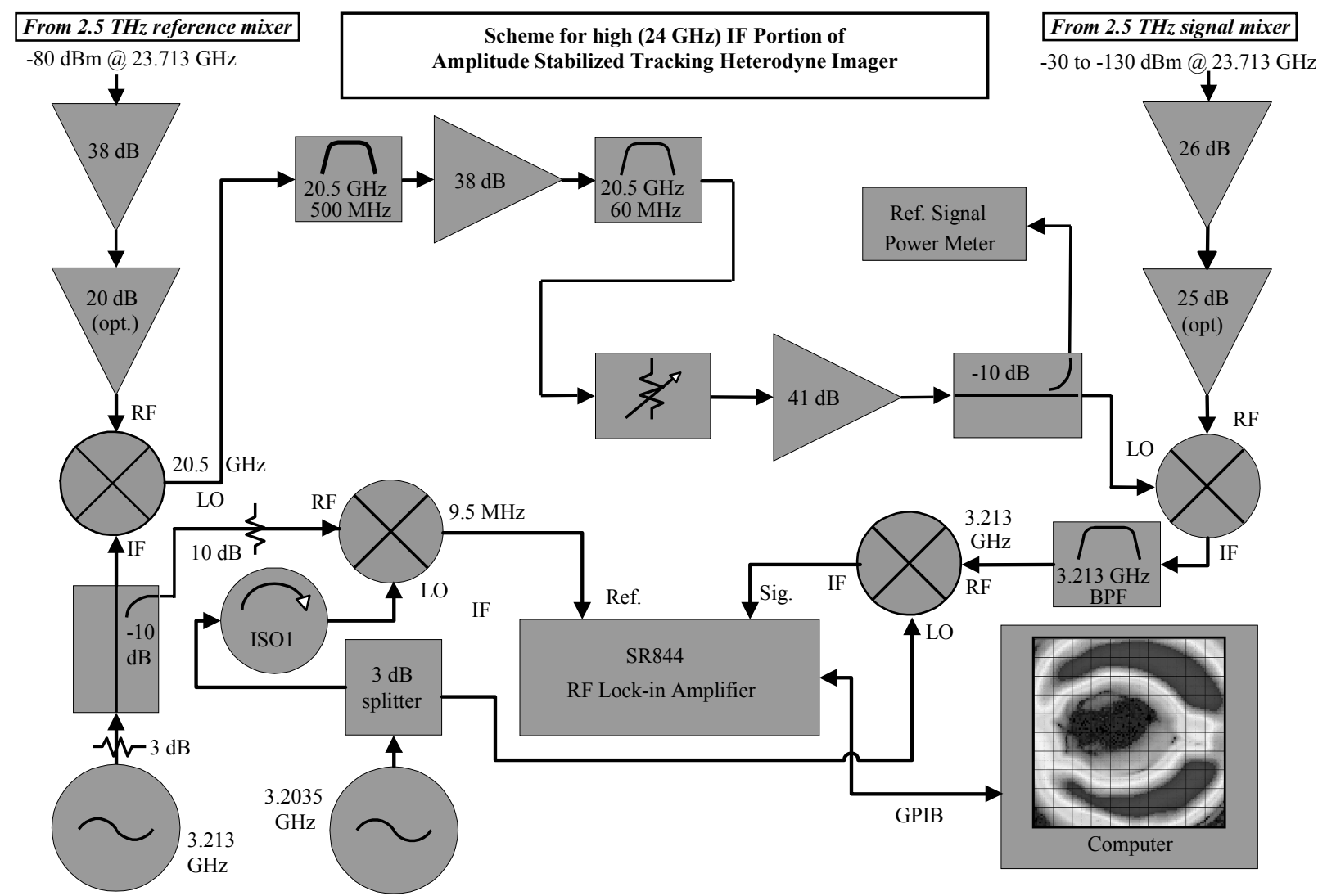

Fig. 7. Intermediate Frequency portion of stabilized heterodyne imager showing tracking amplitude and isolation scheme. 


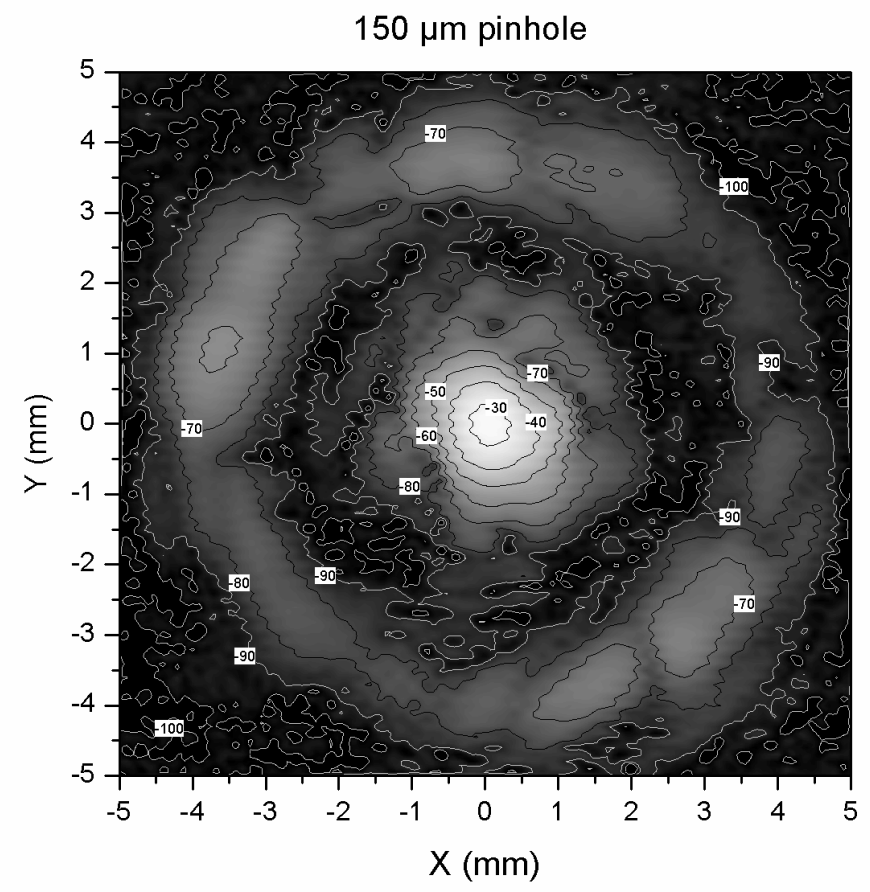

Fig. 8. 2.5 THz laser beam imaged through 150 micron diameter pin hole. Noise floor is $-100 \mathrm{~dB}$ below peak detection level $(0 \mathrm{~dB})$. Transmission maximum through pin hole (center of plot) is $25 \mathrm{~dB}$ below peak detectable power due to large beam diameter. No well defined Airy spot diagram is present because the image is a convolution of the laser beam profile and the diffraction pattern through the pinhole.
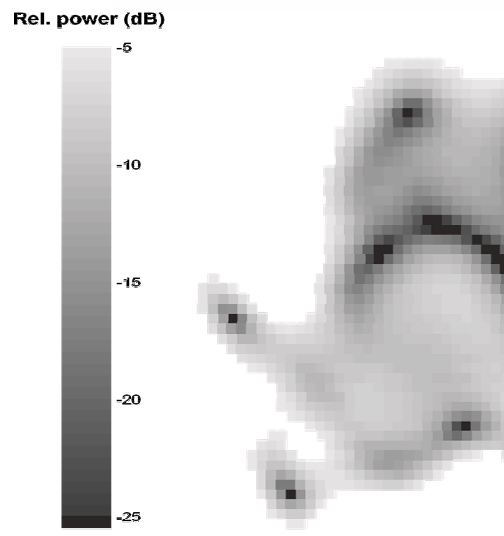

Fig. 9. $2.5 \mathrm{THz}$ magnitude (top left) and phase (bottom right) images of wax mounted lateral brain slices from a shiverer mouse (top right). The slices are approximately $0.5 \mathrm{~mm}$ thick and are not of exactly uniform thickness do to preparation constraints. The magnitude plot has been scaled so that only the brain sample is visible (transparent holder and vignetted edge removed). The phase plot is as measured. The differential absorption that is clearly seen in the $\mathrm{THz}$ images cannot be attributed to any difference in tissue at this time, although further investigations are ongoing with this and other tissue samples. Brain samples from Carol Readhead.
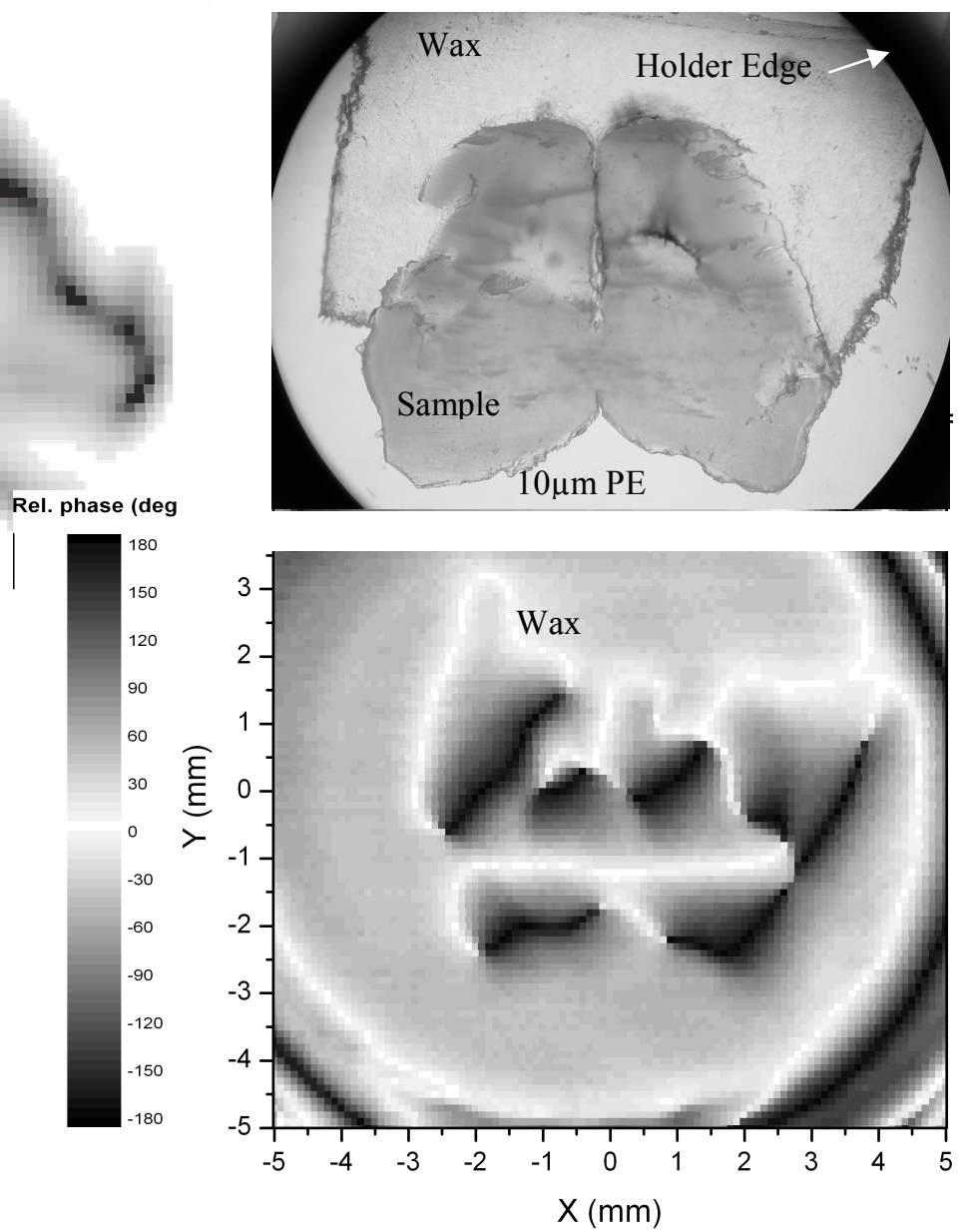